\title{
Analysis on Applying SDN in Wireless Networks from Operation View
}

\author{
W.Q. Sun \\ Institute for Network Sciences and Cyberspace \\ Tsinghua University \\ Tsinghua National Laboratory for Information Science and Technology (TNList) Beijing \\ Beijing, China
}

\author{
H.W. Li \\ Institute for Network Sciences and Cyberspace \\ Tsinghua University \\ Tsinghua National Laboratory for Information Science and \\ Technology (TNList) Beijing \\ Beijing, China
}

\begin{abstract}
Software-defined network (SDN) has received great concern because of its prominent advantages in some aspects such as high flexibility, programmability and dynamic management, etc. Recently, some works begin to apply the concept of SDN in mobile networks to make the networks more open and agile. However, exploiting SDN in wireless networks is still in the initial stages. The potential challenges of applying SDN in an operation wireless network remain unexplored. In this paper, we first summarize related researches and explain the advantages brought by adopting SDN in wireless networks. Then, potential challenges are analyzed base on operation data in a large-scale WLAN. What can be found is that the challenges are either different or more severe than problems of SDN in wired networks because of special characteristics in wireless and mobile environment. In addition, several existing proposals and potential methods concerning on scalability problems are introduced. The analyses in this paper could provide some hints when applying the SDN concept into an operation wireless network.
\end{abstract}

Keywords-SDN; operation; wireless network; challenges

\section{INTRODUCTION}

SDN is an exciting technology that enables innovation in designing and managing networks. Although SDN seems appear suddenly, it is not a new concept but part of a long history of efforts to make networks more programmable. For the past few years, traffic demand is surging and service model is changing, flexible and dynamic network control becomes more urgent to face new trends. SDN decouples control plane and data plane of a network which provides global view, high programmability, and flexible control of the network. Because of those superiorities, OpenFlow, as one SDN protocol, has been tentatively adopted in kinds of scenarios such as enterprise network, data center, and middle-boxes, etc. Certainly, OpenFlow is also adopted to solve problems in mobile networks, since mobile networks have been facing big challenges in these years. For instance, limited resources like radio spectrum have to support surging mobile traffic. Another example is that users are surrounded by dense and chaotic

\author{
J.P. Wu \\ Institute for Network Sciences and Cyberspace \\ Tsinghua University \\ Tsinghua National Laboratory for Information Science and \\ Technology (TNList) Beijing \\ Beijing, China
}

wireless accesses but cannot utilize the networks efficiently and transparently. Traditional network structure is ossified for dealing these problems, while SDN may bring opportunities.

In this article, we try to make an overview on software defined wireless network by explaining related works, presenting probable advantages, and indicating challenges through analyzing data from an operation wireless network. Firstly, we will analyze previous works that introducing SDN into wireless networks. Next, according to related works and urgent problems from the view of operation networks, benefits that may be brought by SDN are illustrated. There are several distinct benefits like heterogeneous accesses integration, unified resource management, network-based mobility support, and economic expenses. Despite all these advantages, SDN also has side-effects like scalability problems that have attracted much attention [1]. Characteristics in real and largescale wireless networks will introduce new factors, thus challenges of SDN might be different from those in wired environment which will be discussed in our paper. Additionally, we also introduce some related works that try to alleviate scalable problems of SDN, but those are not enough for the presented problem resulted by high mobility. The remainder of this paper is organized as follows. After reviewing related works in section 2 , we present advantages of applying SDN concept in wireless networks in section 3. Then the potential challenges are analyzed base on operation data in section 4. Next, in section 5 some proposals including previous ones and future methods to improve scalability are stated. Finally, section 6 concludes the paper.

\section{RELATED WORK}

[2][3][4][5] introduce SDN into cellular networks. Mobi Flow [2] demonstrates a software-defined architecture of EPC which provides maximum flexibility, openness, and programmability to future carriers. Open RAN [3] designs an open and flexible architecture of RAN combing SDN and virtualization. SoftRAN [4] abstracts the radio resources and programs them in a software defined fashion through a 
logically centralized radio access control plane. [5] also identifies several major challenges in cellular data networks that may be solved better by SDN. Towards software-defined WLAN, Odin [6] designs a framework combining SDN and virtual-AP technology. There are some researches considering a more complex scenario, heterogeneous networks. In FP7 project CROWD [7], SDN is identified as a solution to tame extreme density of heterogeneous wireless networks. OpenRoads [8] designs applications upon OpenFlow controller to support mobility in both homogeneous and heterogeneous wireless networks. OpenRadio [9], which is a project of Stanford Networked Systems Group, aims at providing a unified software interface to monitor and program heterogeneous wireless networks.

\section{ADVANTAGES OF WIRELESS SDN}

One basic paradigm of SDN is adopting simple, inexpensive and software-programmable hardware instead of specific hardware. The model could be introduced to cellular network [5] to replace fit-for-purpose devices such as S-GW and P-GW with general hardware. Besides this benefit for expenditure, SDN brings other advantages for wireless and mobile networks. Convergence of heterogeneous wireless accesses: SDN could decouple service definition from the hardware network substrate and provide a unified software interface to monitor and program heterogeneous wireless networks. Unified resource management: SDN could gain a global view of whole wireless accesses and offer flexible method to manage resources. Network-based mobility support: A centralized SDN controller could adapts to the station's movement, signals the devices to set up new flow tables accordingly to the new situation.

\section{Potential Challenges}

To begin with, we present challenges of SDN pointed out by previous works [1][10], most of which are related to scalability. Our analysis takes the OpenFlow network as example, since it is a well-known instantiation of SDN. Controller capacity: A central controller does not scale as the network grows. Therefore, design a scalable structure for control plane is an important and intricate work. Switch flowtable size: OpenFlow rules are stored in a TCAM to support its wildcarding mechanism, and TCAM is an expensive resource, whereas Ethernet forwarding uses a simple hash lookup in a standard memory. [10] exemplifies that the 5406zl switch hardware can support about 1500 OpenFlow rules, while it can support up to 64000 forwarding entries for standard Ethernet switching. Flow setup overhead: Overhead of setting up flows also limits the network scale. A switch has finite bandwidth between their data-plane and control-plane, and finite compute capacity. These issues restrict the rate of flow setups. The best implementations known can set up only a few hundred flows per second [10]. The following content of this section will analyze the influences on SDN brought by some specific characteristics of real and large-scale wireless networks.

\section{A. Control of Wireless Accesses}

In an SDN wired network, a centralized controller monitors switches and sets forwarding table on switches for flows. Besides these tasks, a controller in software-defined wireless network has to responsible for more works. Management of access points and base stations in wireless accesses is different from that of switches. That is why there are control elements for them in traditional networks, like WLC (wireless LAN controller) for thin APs in WLAN and RNC for base stations. Since SDN decouples control plane and data plane, control function of APs and base stations should be defined by centralized software. For instance, on-demand spectrum allocation for APs and base stations may be coordinated globally by central controller. However, control plane separation from APs and base stations requires meticulous design to avoid putting a heavy burden on the controller and introducing high delay. Possibly, APs and base stations have to keep part control locally. Hence, improved architecture and new protocols are demanded to build software-defined wireless networks.

\section{B. Multiple Interfaces \& Multiple Kinds of Service}

In current days, mobile stations generally have multiple interfaces to access heterogeneous networks, such as $3 \mathrm{G}, 4 \mathrm{G}$ and WLAN, etc. And it is believed that interfaces of one mobile station will become more in the future. To utilize resources efficiently and get high-quality services, mobile stations tend to use multiple interfaces simultaneously, as we can found that research on multipath transfer has attracted much attention. Moreover, multiple kinds of services (voice, IM, file transfer, multimedia, etc) may run on multiple interfaces concurrently. All that means there will be much more flows in wireless networks than that in wired networks. Flows of different kinds or interfaces of one station are possibly required to be distinguished in the networks. As presented in the beginning part of this section, an OpenFlow switch can support several thousands of flow rules, affected by multiple interfaces and multiple kinds of flows, even only several hundreds of mobile stations could be supported by one switch. As illustrated, not only the control plane, but also the data plane in wireless networks is facing the challenge of scalability.

\section{High Mobility}

High frequency of user movement is a distinct character in wireless and mobile networks. With development of wireless network and widespread of intelligent terminals, user mobility characteristics has changed considerably. About 10 years ago, measurements [11] in three WLANs showed that most users stayed at a certain location for most of the time and visited less than 3, 7, 10 APs within one day respectively in the three networks. It can be inferred that most of stations in at that time were laptops. And few people would like to keep walking around with their bulky laptops. But today, most people bring at least one smart terminal with them everywhere. Then there will be much more mobile events in today's network. Operation data of our campus WLAN are fetched and analyzed to show the mobility characteristics. In one day, more than 46000 devices appeared in the network. The devices are into several classes according to MAC address. As shown in figure 1(a), although many MAC addresses cannot be recognized, we can still found that PDAs including phones and tablets account for $58 \%$ of the whole stations. And specific kinds of PDAs are depicted in figure 1(b). Not surprisingly, smart phones and portable terminals rather than laptops are 
predominant devices in current wireless network. The frequency and range of user movement are also looked into and shown in figure 2. Handoffs (changing associated AP) of stations are calculated to observe mobile frequency. And there are 11 WLAN controllers taking charge of different areas of the campus wireless network. A handoff across controllers means that the mobile user roams to the managing realm of a different controller. Handoffs across controllers can be used as some hints to understand the mobile range. Figure 2 reveals that about $90 \%$ of the laptops experience less than 5 handoffs (line of "Laptop(all)"), whereas the corresponding percent of PDAs that perform less than 5 handoffs is about $68 \%$ (line of "PDA(all)"). The result indicates that PDAs perform much more mobile events than laptops. As to the mobile range, PDAs also experience more handoffs across controllers.

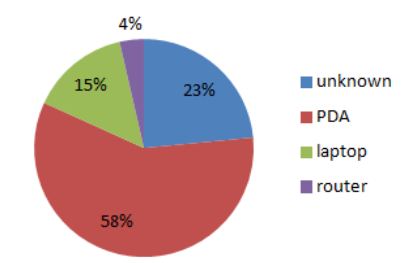

(a) Ratios of different kinds of stations

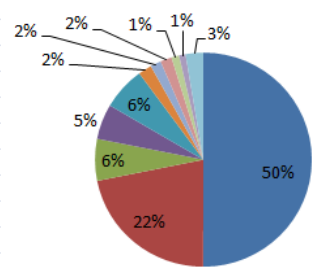

(b) Ratios of different kinds of PDAs
FIGURE I. STATICS OF STATIONS IN THE CAMPUS WIRELESS NETWORK.

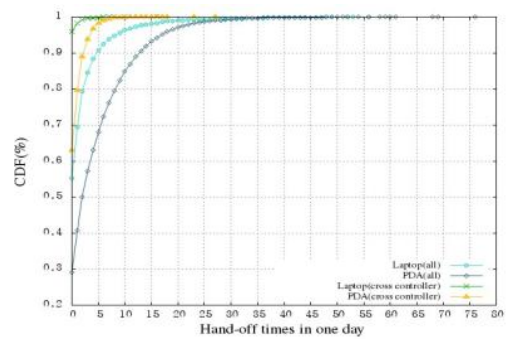

FIGURE II. CDF OF HAND-OFF TIMES

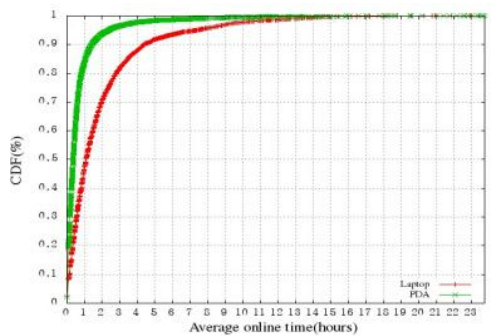

FIGURE III. CDF OF AVERAGE ONLINE TIME

Figure 3 presents the average online time of laptops and PDAs. Average online periods of about $50 \%$ of PDAs are less than 20 minutes. Average online periods of PDAs are obviously shorter than those of laptops. In one word, according to our observation, most stations in current wireless network are portable terminals which move frequently and keep online for short periods. These mobility characteristics may bring significant influences on software-defined wireless networks. When a station moves to a new AP, the SDN controller has to calculate and set new forwarding paths for flows of the station. In the process, certain signaling packets are generated and many switches may be evolved. We give an example to illustrate overheads brought by user mobility. A simple topology is shown in following figure 4. MN moves from AP1 to AP2 while sending data to $\mathrm{CN}$. Then the forwarding path changes from (AP1->Sw1->Sw4->Sw3,$>$ AP3) to (AP2->Sw2->Sw4->Sw3->AP3). We analyze the signaling overheads based on the observation of flow-setup process in Mininet. Signaling packets sent or received by the devices to realize above path resetting are listed in table 1 . In such a simple topology, for supporting one handoff of a unidirectional flow on a station, 8 signaling packets are generated and 5 devices are involved. It can be imagined that in a large-scale wireless network with complex topology, many mobile users with multiple flows will bring a large amount of overhead. Furthermore, data packets are usually few while the station is moving, which means that many - Applesignaling packets are generated to support only a few data - Samsung pâckets. Compared with the moving event, "ping-pong" effect Huawiesults in worse consequence. For instance, assuming that MN sonyin figure 4 can receives wireless signals from both AP1 and $=$ Mokia $A P 2$, and then it may perform handoffs between the two APs

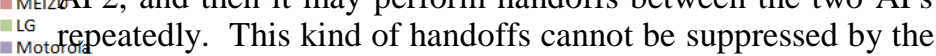
Lenorpetwork since it depends on signal strength and driver implementation. Unfortunately, "ping-pong" effects are easy to find in the campus WLAN. As shown in figure 2, some stations perform nearly 80 handoffs because of "ping-pong" effect. To sum up, the high mobility of stations will cause a large amount of signaling overheads in software defined wireless network. As presented before, flow-setup overhead is one of the main limitations for SDN scalability. Therefore, schemes to support mobility in SDN should be optimized to reduce overheads.

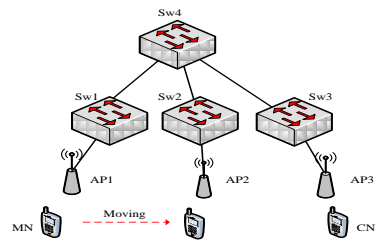

FIGURE IV. A SIMPLE MOBILITY SCENARIO

TABLE I. SIGNAL PACKETS TO SUPPORT A MOVING FLOW

\begin{tabular}{|l|l|l|l|l|}
\hline & $\begin{array}{c}\text { Packet_in } \\
\text { (send) }\end{array}$ & $\begin{array}{l}\text { Packet_out Flow_modify } \\
\text { (receive) }\end{array}$ & $\begin{array}{c}\text { Flow_remove } \\
\text { (receive) }\end{array}$ & (receive) \\
\hline Sw1 & 0 & 0 & 0 & 1 \\
\hline Sw2 & 1 & 1 & 1 & 0 \\
\hline Sw4 & 0 & 0 & 1 & 0 \\
\hline AP1 & 0 & 0 & 0 & 1 \\
\hline AP2 & 1 & 1 & 0 & 0 \\
\hline
\end{tabular}

\section{SOLUTIONS TOWARDS SCALABILITY PROBLEMS}

As analyzed in last section, scalability is a common recognized problem in SDN and the problem will become more severe in wireless and mobile environment. Previous research [10][12][13][14] have brought out some techniques to alleviate scalability problems. Those solutions could make SDN structure more scalable, but there are still unsolved problems, such as the overhead of setting up paths for flows 
with frequent handoffs. Even if the Kandoo framework is applied to relieve overhead on controllers, frequent handoffs also generate much overhead on switches which has been discussed before. A potential solution is to make switches support some local actions without involving control plane. For instance, if one flow changes in-port on a switch because of handoffs, action of flow modification on that switch may be processed by switches not the controller. In this way, the control overhead can be cut down drastically. It is only a possible method which still requires detail consideration and will be discussed in the future.

\section{CONCLUSION}

In this paper, we discuss several possible advantages, challenges of embracing SDN in wireless and mobile environment. To sum up, there may be unexpected challenges and complexity when applying SDN in operation wireless networks. Mining the operation data and analyze user behaviors could provide some clues for finding and solving problems.

\section{REFERENCES}

[1] Yeganeh S H, Tootoonchian A, Ganjali Y. On scalability of softwaredefined networking[J]. Communications Magazine, IEEE, 2013, 51(2): 136-141

[2] PENTIKOUSIS K, WANG Y, HU W. Mobileflow: Toward softwaredefined mobile networks [J]. Communications Magazine, IEEE, 2013, 51(7).

[3] YANG, MAO, et al. OpenRAN: a software-defined ran architecture via virtualization[C], Proceedings of the ACM SIGCOMM 2013 conference on SIGCOMM,2013,pp549-550

[4] GUDIPATI, ADITYA, et al. SoftRAN: Software Defined Radio Access Network[C], Proceedings of workshop on Hot topics in software defined networks, ACM,2013

[5] LI L E, MAO Z M, and Rexford J, Toward software-defined cellular networks[C], Software Defined Networking (EWSDN), 2012 European Workshop on,pp7-12.

[6] SURESH, LALITH, et al. Towards programmable enterprise WLANs with Odin[C], Proceedings of the first workshop on Hot topics in software defined networks, ACM, 2012, pp115-120.

[7] De La Oliva A, Morelli A, Mancuso V, et al. Denser networks for the Future Internet, the CROWD approach[M]//Mobile Networks and Management. Springer Berlin Heidelberg, 2013: 28-41.

[8] Yap K K, Kobayashi M, Sherwood R, et al. OpenRoads: Empowering research in mobile networks[J]. ACM SIGCOMM Computer Communication Review, 2010, 40(1): 125-126.

[9] Bansal M, Mehlman J, Katti S, et al. Openradio: a programmable wireless dataplane[C]//Proceedings of the first workshop on Hot topics in software defined networks. ACM, 2012: 109-114.

[10]Curtis A R, Mogul J C, Tourrilhes J, et al. DevoFlow: scaling flow management for high-performance networks[C]//ACM SIGCOMM Computer Communication Review. ACM, 2011, 41(4): 254-265.

[11]Balazinska M, Castro P. Characterizing mobility and network usage in a corporate wireless local-area network[C]/Proceedings of the 1 st international conference on Mobile systems, applications and services. ACM, 2003: 303-316.

[12]Tootoonchian A, Ganjali Y. Hyperflow: a distributed control plane for openflow[C]//Proceedings of the 2010 internet network management conference on Research on enterprise networking. USENIX Association, 2010: 3-3.

[13]Jin X, Li L E, Vanbever L, et al. SoftCell: scalable and flexible cellular core network architecture[C]//Proceedings of the ninth ACM conference on Emerging networking experiments and technologies. ACM, 2013.
[14]Hassas Yeganeh S, Ganjali Y. Kandoo: a framework for efficient and scalable offloading of control applications[C]/Proceedings of the first workshop on Hot topics in software defined networks. ACM, 2012 